\title{
Pharmacokinetic and pharmacodynamic profiles of recombinant human erythropoietin-loaded poly(lactic-co-glycolic acid) microspheres in rats
}

\author{
Xiang-lian ZHOU ${ }^{1}$, Jin-tian $\mathrm{HE}^{1, *}$, Hui-juan $\mathrm{DU}^{1}$, Yang-yang $\mathrm{FAN}^{1}$, Ying $\mathrm{WANG}^{2}$, Hong-xia ZHANG ${ }^{2}$, Yang JIANG ${ }^{2}$ \\ ${ }^{1}$ College of Life Science, Hebei Normal University, Shijiazhuang 050016, China; ${ }^{2}$ NCPC GeneTech Biotechnology Development Co, Ltd, \\ Shijiazhuang 050000, China
}

Aim: To characterize the pharmacokinetic and pharmacodynamic profiles of the recombinant human erythropoietin (rhEPO)-loaded poly(lactic-co-glycolic acid) (PLGA) microspheres in rats.

Methods: The rhEPO-loaded microspheres were prepared using a solid-in-oil-in-water emulsion method. Pharmacokinetics and pharmacodynamics of the rhEPO-loaded microspheres were evaluated in male Sprague-Dawley rats. The serum rhEPO level was determined with ELISA. The level of anti-rhEPO antibody in the serum was measured to assess the immunogenicity of rhEPO released from the microspheres.

Results: rhEPO was almost completely released from the PLGA microspheres in vitro, following zero-order release kinetics over approximately $30 \mathrm{~d}$. After intramuscular injection (10 000 or $30000 \mathrm{IU}$ rhEPO/kg) in the rats, the serum rhEPO concentration reached maximum levels on $\mathrm{d} 1$, then decreased gradually and was maintained at nearly steady levels for approximately 4 weeks. Furthermore, the release of rhEPO from the PLGA microspheres was found to be controlled mainly by a dissolution/diffusion mechanism. A good linear correlation $\left(R^{2}=0.98\right)$ was obtained between the in vitro and in vivo release data. A single intramuscular injection of the rhEPOloaded PLGA microspheres (10 000 or $30000 \mathrm{IU}$ rhEPO/kg) in the rats resulted in elevated hemoglobin and red blood cell concentrations for more than $28 \mathrm{~d}$. Moreover, the immunogenicity of rhEPO released from the PLGA microspheres was comparable with that of the unencapsulated rhEPO.

Conclusion: The results prove the feasibility of using the PLGA-based microspheres to deliver rhEPO for approximately 1 month.

Keywords: recombinant human erythropoietin; poly(lactic-co-glycolic acid); microspheres; pharmacokinetics; pharmacodynamics

Acta Pharmacologica Sinica (2012) 33: 137-144; doi: 10.1038/aps.2011.157; published online 5 Dec 2011

\section{Introduction}

Erythropoietin (EPO) is a hormone primarily produced by kidney cells and is the main regulator of red blood cell (RBC) production in mammals. Recombinant EPO is used to treat anemia resulting from renal failure, zidovudine treatment for HIV infection, bone marrow transplantation, and cancer chemotherapy ${ }^{[1-3]}$. Recombinant EPO is usually administered via two or three intravenous or subcutaneous injections per week for several years. Unfortunately, no patient-friendly administration routes exist other than these parenteral routes. To improve patient compliance and therapeutic efficacy, a sustained-release delivery system that permits EPO administration only once or twice a month is desirable.

\footnotetext{
* To whom correspondence should be addressed.

E-mail he_jintian@yahoo.com

Received 2011-07-08 Accepted 2011-10-19
}

Injectable, sustained-release biodegradable microspheres could control drug release for several days or even several months. The development of biodegradable polymeric microspheres as carriers has become a promising way to overcome problems with drug administration ${ }^{[4,5]}$. Some poly $(D, L$-lacticco-glycolic acid) (PLGA) depot products containing peptide hormones or other drugs have become commercially available, including LHRH agonists, somatostatin derivatives and estra$\mathrm{diol}^{[4]}$. Over the last decade, many attempts have been made to develop biodegradable microsphere systems for rhEPO. Morlock et al developed PLGA and PLGA-PEO-PLGA triblock copolymer microspheres using a water-in-oil-in-water $(\mathrm{w} / \mathrm{o} / \mathrm{w})$, double-emulsion micro-encapsulation process ${ }^{[6-8]}$. EPO was continuously released from the microspheres for up to 2 weeks in vitro. However, rhEPO (recombinant human erythropoietin) was susceptible to the micro-encapsulation processes and easily aggregated. The amount of aggregated 
rhEPO within the microspheres prepared using the $\mathrm{w} / \mathrm{o} / \mathrm{w}$ double-emulsion technique was higher than safety standards allow ( $\leq 2 \%$ ) (for a solution from EPO) ${ }^{[8]}$. Geng et al reported a novel method to prepare erythropoietin-loaded PLGA microspheres ${ }^{[9]}$. EPO was first formulated with dextran to form EPO-dextran glassy particles. These particles were subsequently encapsulated into PLGA microspheres using a solidin-oil-in-water (s/o/w) emulsion method. The stability of EPO was effectively preserved during this preparation process (aggregation of $\mathrm{EPO}<2 \%$ ). An in vitro release study showed that EPO could be released from the composite PLGA microspheres in a sustained-release manner up to $60 \mathrm{~d}$. However, the in vivo efficacy of EPO was maintained for only approximately $30 \mathrm{~d}$. To achieve optimum therapeutic efficacy for the rhEPO-loaded microspheres, the relationship between in vitro drug release and in vivo pharmacokinetics and pharmacodynamics should be well characterized. However, the in vivo pharmacokinetics and pharmacodynamics of EPO-loaded microspheres has rarely been investigated in detail until now.

In the present study, PLGA microspheres loaded with rhEPO were fabricated by an s/o/w emulsion solvent evaporation method. The in vitro release kinetics, in vivo pharmacokinetics and pharmacodynamics of the rhEPO-loaded PLGA microspheres were evaluated. The correlation between in vitro release kinetics and in vivo pharmacokinetics of the microspheres was examined. In addition, the acute toxicity and immunogenicity of the rhEPO-loaded microspheres were investigated in rats.

\section{Materials and methods Materials}

The rhEPO solution was obtained from NCPC GeneTech Biotechnology Development Co, Ltd (Shijiazhuang, China). Polyethylene glycol (PEG) with an average molecular weight of 6000 Da was purchased from Sigma (St Louis, MO, USA). Polyvinyl alcohol (PVA) with a molecular weight range of 31000-50000 Da was obtained from Aldrich Chemical Company Inc (USA). PLGA was purchased from the Ji-nan Daigang Biomaterial Co, Ltd (Ji-nan, China). PLGA is a copolymer with $D L$-lactide/glycolide ratio of 75:25 and an average molecular weight of approximately $23 \mathrm{kDa}$. Human serum albumin (HSA) was obtained from Shanghai RAAS Blood Products Co, Ltd (Shanghai, China). All other chemicals used were of analytical grade.

\section{Preparation of the rhEPO-HSA microparticles}

The rhEPO-HAS microparticles were prepared using a modified freezing-induced phase separation method ${ }^{[10]}$. In brief, a solution containing $0.1 \% \operatorname{rhEPO}(w / v), 1 \% \operatorname{HSA}(w / v), 5 \%$ PEG $(w / v)$ and $0.02 \mathrm{~mol} / \mathrm{L}$ sodium phosphate was frozen at $-80^{\circ} \mathrm{C}$ overnight. Subsequently, the solution was lyophilized using a Christ ALPHA 1-2 plus freeze-dryer at a pressure of $5.0 \times 10^{-3} \mathrm{~Pa}$ for $20 \mathrm{~h}$. The lyophilized powders were dispersed in $5 \mathrm{~mL}$ of dichloromethane with high-speed agitation. To remove the PEG within the particles, the mixtures were centrifuged at $8000 \mathrm{r} / \mathrm{min}$ for $10 \mathrm{~min}$. This operation was repeated three times. The final precipitates were dried overnight under a vacuum.

\section{Preparation of the rhEPO-loaded PLGA microspheres}

The rhEPO-loaded PLGA microspheres were prepared using a modified s/o/w method ${ }^{[11]}$. Briefly, $10 \mathrm{mg}$ of the rhEPO-HSA mixture microparticles was suspended in $2.5 \mathrm{~mL}$ of dichloromethane solution containing $180 \mathrm{mg} / \mathrm{mL}$ of PLGA. An IKA T25 ultra-turrax homogenizer was used at $20000 \mathrm{r} / \mathrm{min}$ for 1.5 min. The resulting solid-in-oil (s/o) suspension was mixed with $75 \mathrm{~mL}$ of $2 \%(w / v)$ aqueous PVA solution containing $5 \%$ $(w / v) \mathrm{NaCl}$. The mixture was then homogenized at $600 \mathrm{r} / \mathrm{min}$ for $1 \mathrm{~min}$ to form the s/o/w emulsion. For solvent extraction, the s/o/w emulsion was immediately diluted with $225 \mathrm{~mL}$ of $20 \mathrm{mmol} / \mathrm{L}$ phosphate buffer (PB; pH 7.4) containing 5\% $\mathrm{NaCl}$. The emulsion was stirred with a magnetic stirrer at 300 $\mathrm{r} / \mathrm{min}$ for $6 \mathrm{~h}$. The resulting rhEPO-loaded microspheres were collected by filtration and washed three times with distilled water to remove the PVA and $\mathrm{NaCl}$. The microspheres were then lyophilized using a Christ ALPHA 1-2 plus freeze-dryer operating at a pressure of $5.0 \times 10^{-3} \mathrm{~Pa}$ overnight. The samples were stored at $-20^{\circ} \mathrm{C}$.

\section{Microsphere characterization and determination of drug encapsulation efficiency}

The rhEPO-HSA mixture microparticles and the rhEPO-loaded PLGA microspheres were examined for morphology and size by scanning electron microscopy (SEM) using a Hitachi S-520. The rhEPO-HSA mixture microparticles and the rhEPOloaded PLGA microspheres were mounted onto metal stubs using double-sided adhesive tape and vacuum coated with a thin layer of gold. Then, the samples were examined by SEM. One hundred rhEPO-HSA mixture microparticles or PLGA microspheres were randomly selected and measured in diameter; the results were reported as an average value.

The protein content in the microspheres was determined using a previously described extraction method ${ }^{[12]}$. Thirty milligrams of dried microspheres were dissolved in methylene chloride and centrifuged at $10000 \mathrm{r} / \mathrm{min}$ for $15 \mathrm{~min}$ to remove the polymer solution. The remaining protein pellet was dissolved in $0.2 \mathrm{~mL}$ of $20 \mathrm{mmol} / \mathrm{L} \mathrm{PB}$ (pH 7.4). The rhEPO concentration was determined using a Quantikine in vitro diagnostic rhEPO ELISA kit purchased from R\&D Systems (Minneapolis, MN, USA). The protein encapsulation efficiency in the microspheres was calculated as the ratio of actual to theoretical protein loadings.

\section{In vitro release of proteins from PLGA microspheres}

Thirty milligrams of dried microspheres were suspended in 1 $\mathrm{mL}$ of $0.02 \mathrm{~mol} / \mathrm{L}$ phosphate buffered saline (PBS, $\mathrm{pH} 7.4$ ) containing $0.05 \%(w / v)$ sodium azide. The samples were agitated in a rotating bottle apparatus at $50 \mathrm{r} / \mathrm{min}$ at $37^{\circ} \mathrm{C}$. Samples of $1 \mathrm{~mL}$ were removed at the following sampling times after centrifugation at $3000 \mathrm{r} / \mathrm{min}$ for $10 \mathrm{~min}: 0,1,3,5,8,11,14,17$, $21,25,29$, and $33 \mathrm{~d}$. The removed medium was immediately replaced with the same volume of fresh PBS solution. The 
rhEPO content in the supernatant was determined using a Quantikine in vitro diagnostic rhEPO ELISA kit purchased from R\&D Systems (Minneapolis, MN, USA). The amount of rhEPO released within $24 \mathrm{~h}$ was defined as the initial burst.

Pharmacokinetics of the rhEPO-loaded PLGA microspheres in rats In vivo release of the rhEPO-loaded PLGA microspheres was evaluated in male SD rats (male, 180-210 g, Grade II, Certificate No 06057) purchased from the Experimental Animal Center of Hebei Province in China. The rats were housed under conventional laboratory conditions in a room maintained at $24 \pm 1^{\circ} \mathrm{C}$ and fed commercial rat food and water ad libitum. The rats were randomly divided into four groups of six rats each. One group was used for intramuscular injection of rhEPO solution at a dose of $30000 \mathrm{IU} / \mathrm{kg}$, the second and the third groups were used for intramuscular injection of the microspheres at a dose of 10000 or $30000 \mathrm{IU} / \mathrm{kg}$ of rhEPO, and the fourth group was used for intramuscular injection of the equivalent amount of blank microspheres (without rhEPO) as a control. The rhEPO-loaded microspheres were pre-suspended in the diluent containing $2 \%$ carboxymethylcellulose sodium and $0.9 \%(w / v) \mathrm{NaCl}$. One milliliter of the formulations was injected at multiple points into the gastrocnemius muscle of the hind legs. After injection, $0.4 \mathrm{~mL}$ of blood was withdrawn from the retro-orbital sinus through the eye canthus of anesthetized rats at $0.5,1,3,6,9,12,16,20$, and $24 \mathrm{~h}$ after injection of the rhEPO solution and at 1, 4, 8, 13, 18, $23,28,33$, and $38 \mathrm{~d}$ after injection of the microsphere formulations. The obtained blood samples were centrifuged at 1500 $\mathrm{r} / \mathrm{min}$ for $15 \mathrm{~min}$. Approximately $0.2 \mathrm{~mL}$ of plasma samples was obtained and stored at $-40^{\circ} \mathrm{C}$. All animal studies were performed in Hebei Normal University in accordance with Institutional Animal Care and Use Committee guidelines.

The content of rhEPO in plasma samples was determined using a Quantikine in vitro diagnostic rhEPO ELISA kit purchased from R\&D Systems (Minneapolis, MN, USA). Pharmacokinetic parameters were calculated directly from the plasma concentration versus time plot ${ }^{[13]}$. The area under the curve (AUC) was calculated by the trapezoidal method. The apparent elimination rate constants $\left(K_{\mathrm{e}}\right)$ were determined from the semi-logarithmic plot of plasma concentration versus time. $k$ was calculated from the terminal linear portion of the curve using linear regression analysis ${ }^{[13]}$. The terminal elimination half-lives $\left(t_{1 / 2}\right)$ were calculated by dividing 0.693 by the elimination rate constant ${ }^{[13]}$.

\section{Pharmacodynamics of the rhEPO-loaded PLGA microspheres in} rats

The pharmacodynamics of the rhEPO-loaded PLGA microspheres were also evaluated in male SD rats. The rats were randomly divided into 4 groups with 6 rats in each group. The drug administration method was the same as for the pharmacokinetic study. After intramuscular injection, $20 \mu \mathrm{L}$ of blood was collected from the rats (at the tail vein) at each sampling time after starting the treatment. The blood sample was immediately mixed with $0.5 \mathrm{~mL}$ of dilution buffer provided by the manufacturer. The hemoglobin (HGB) concentration and red blood cell number (RBC) in the blood samples was determined using an XE-2100 automated hematology analyzer (Sysmex, Japan).

\section{In vivo polymeric degradation in rats}

To determine the sites of microspheres in rats, the injection site was marked with 3\%-5\% picric acid solution. At designated time points, the rats were sacrificed in an induction chamber filled with ether, the muscles at the injection site were cut, and the microspheres were removed. The degradation of the rhEPO-loaded PLGA microspheres in rats was observed with scanning electron microscopy (SEM, Hitachi S-520).

\section{Acute toxicity test in rats}

Forty SD rats (180-210 g, Grade II, Certificate No 06057), 20 males and 20 females, were used for the acute toxicity test. According to the maximum dosage method, the available maximum dose of the rhEPO-loaded PLGA microspheres was $2000 \mathrm{mg} / \mathrm{kg}$, which is equivalent to approximately 50 times the normal clinical dose for humans. The lower doses were $500 \mathrm{mg} / \mathrm{kg}, 50 \mathrm{mg} / \mathrm{kg}$, and $5 \mathrm{mg} / \mathrm{kg}$, respectively and the medium was used as a control. After intramuscular injection into the hind limbs, the rats were observed for acute reactions including tachypnea, restlessness and stupor. In addition, the rats were weighed daily throughout the two-week observation period.

\section{Detection of anti-rhEPO antibody}

To examine the immune response to the rhEPO-loaded PLGA microspheres, $2000 \mathrm{IU} / \mathrm{kg}$ rhEPO in solution, $2000 \mathrm{IU} / \mathrm{kg}$ rhEPO in microsphere form, $2000 \mathrm{IU} / \mathrm{kg}$ rhEPO in denatured HSA-rhEPO mixture (heated at $100^{\circ} \mathrm{C}$ for $10 \mathrm{~min}$ ) and the equivalent amount of blank microspheres (without rhEPO) were injected intramuscularly into SD rats (5 rats per group). The amount of anti-rhEPO antibody in the blood samples taken at the 6th week was analyzed by ELISA. Briefly, polystyrene microtiter plates were coated overnight at $4{ }^{\circ} \mathrm{C}$ with $100 \mu \mathrm{L}$ of $0.1 \mathrm{mg} / \mathrm{mL}$ rhEPO in coating buffer $(50 \mathrm{mmol} / \mathrm{L}$ sodium carbonate buffer $\mathrm{pH}$ 9.6) and rinsed with PBST (0.01 mol/L PBS at pH 7.4 containing $0.1 \%$ Tween 20). After blocking with $5 \%(w / v)$ dried skimmed milk powder at $37^{\circ} \mathrm{C}$ for $2 \mathrm{~h}$, the microtiter plates were washed three times with PBST. One hundred microliters of the diluted serum samples $(1 / 200)$ were loaded and incubated at $37^{\circ} \mathrm{C}$ for $1 \mathrm{~h}$. After the plates were washed three times with PBST, $100 \mu \mathrm{L}$ of horseradish peroxidase-labeled rabbit anti-rat polyclonal immunoglobulin diluted 1:2000 was added and incubated with for $1 \mathrm{~h}$ at room temperature. The plate was again washed three times with PBST, and the bound enzyme conjugate was detected by adding $100 \mu \mathrm{L}$ of 3,3,5,5-tetramethylbenzidine (substrate) to each well. The reaction was stopped 10-20 min later using $50 \mu \mathrm{L}$ of $2 \mathrm{~mol} / \mathrm{L} \mathrm{H}_{2} \mathrm{SO}_{4}$. The absorbance at $450 \mathrm{~nm}$ was measured on a Bio-Rad 550 microplate reader. 


\section{Statistical analysis}

The differences between two groups were evaluated statistically using analysis of variance (ANOVA) followed by the Newman-Keuls test. A $P$ value of $<0.05$ was considered statistically significant. All data analysis was performed with the Statistica statistical software package (version 6.0, StatSoft Inc, Tulsa, OK, USA).

\section{Results}

Characterization of the rhEPO-HSA mixture microparticles and the rhEPO-loaded PLGA microspheres

Figures $1 \mathrm{~A}$ and $1 \mathrm{~B}$ show SEM images of the rhEPO-HAS microparticles and the rhEPO-loaded PLGA microspheres, respectively. The rhEPO-HSA microparticles possessed a spherical shape, smooth surface and diameters ranging between 200 and $600 \mathrm{~nm}$. The rhEPO-loaded PLGA microspheres showed a spherical, smooth, nonporous and solid morphology, with sizes ranging between 40 and $100 \mu \mathrm{m}$ in diameter. The rhEPO encapsulation efficiency and actual drug loading were $85.3 \%$ and $0.21 \%$, respectively.
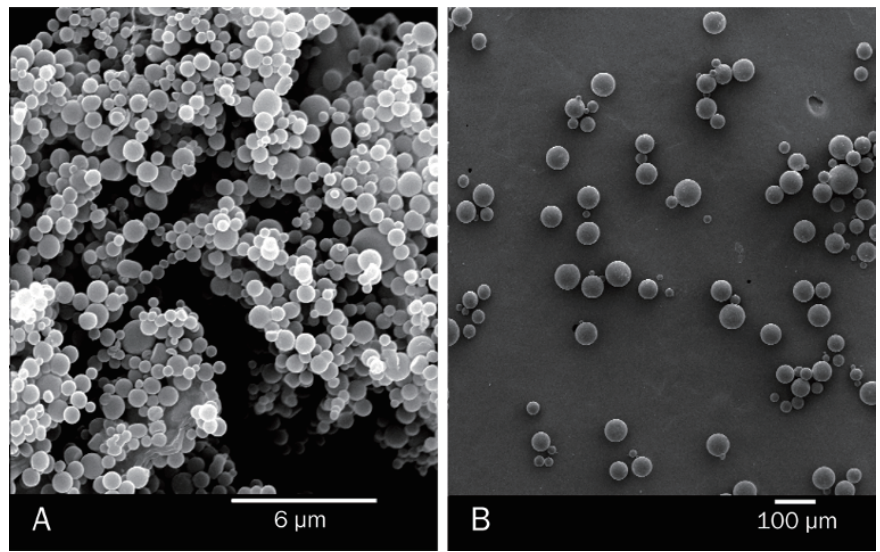

Figure 1. Scanning electron microscopic images of freeze-dried rhEPOHSA mixture microparticles and rhEPO-loaded PLGA microspheres. (A) rhEPO-HSA mixture microparticles; (B) rhEPO-loaded PLGA microspheres.

\section{In vitro drug release}

The in vitro release profile of rhEPO from the PLGA microspheres is presented in Figure 2. The in vitro release profile exhibited a biphasic pattern. The first phase was an initial burst release of approximately $17 \%$ of the loaded rhEPO, which occurred within one day. After the initial burst, rhEPO release was sustained, following zero-order release kinetics for approximately $30 \mathrm{~d}$. The linear regression equation was inserted into Figure 2. A good linear regression correlation was made between time and the percentage of drug released in PBS at $37^{\circ} \mathrm{C}$; the correlation coefficient was greater than 0.98 , indicating that ideal zero-order kinetics was obtained. Furthermore, rhEPO was nearly completely released from the PLGA microspheres after $33 \mathrm{~d}$.

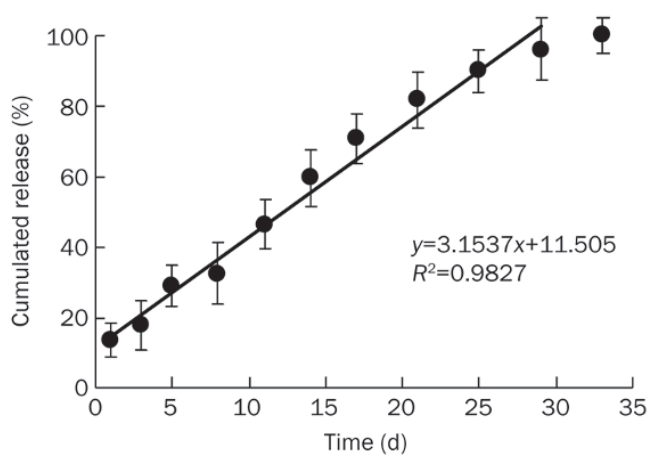

Figure 2. In vitro cumulative release profiles of $\operatorname{rhEPO}(\bullet)$ from the rhEPOloaded PLGA microsphere.

\section{In vivo pharmacokinetic studies}

Figure 3A shows the in vivo rhEPO concentration-time profile after intramuscular injection of rhEPO solution in rats. The peak plasma concentration $\left(C_{\max }\right)$ for the rhEPO solution was $17.85 \mathrm{mIU} / \mathrm{mL}$, and the time to attain the peak concentration $\left(T_{\max }\right)$ was $0.3 \mathrm{~h}$. The rapid decrease in rhEPO concentration indicated that the elimination of rhEPO was rapid. The fast decrease in rhEPO concentration suggested that frequent injections are needed to maintain a constant plasma rhEPO level. After analyzing the concentration-time profiles, the apparent elimination rate constant $\left(K_{\mathrm{e}}\right)$ and terminal elimination halflife $\left(t_{1 / 2}\right)$ after intramuscular injection were determined to be $0.75 \mathrm{~h}^{-1}$ and $9.6 \mathrm{~h}$, respectively (Table 1$)$. These results are consistent with those from a previous report ${ }^{[3]}$.

Figure 3B shows the rhEPO plasma concentration versus time profile after intramuscular injection of the rhEPO-loaded PLGA microspheres. The pharmacokinetic profiles for all

Table 1. Pharmacokinetic parameters for the rhEPO solution and the rhEPO-loaded microspheres (MS) after intramuscular injection in SD rats. Mean \pm SD. $n=3$.

\begin{tabular}{lrrr}
\hline \multicolumn{1}{c}{ Samples } & $t_{1 / 2}(\mathrm{~d})$ & $k_{\mathrm{e}}\left(\mathrm{d}^{-1}\right)$ & AUC $_{0-\mathrm{t}}(\mathrm{mlUd} / \mathrm{mL})^{\mathrm{a}}$ \\
& & & \multicolumn{1}{c}{ Relative } \\
bioavailability $(\%)$
\end{tabular}

${ }^{a}$ The AUC was calculated from 0 to $24 \mathrm{~h}$ for the rhEPO solution and from 0 to $33 \mathrm{~d}$ for the rhEPO-loaded microspheres. 

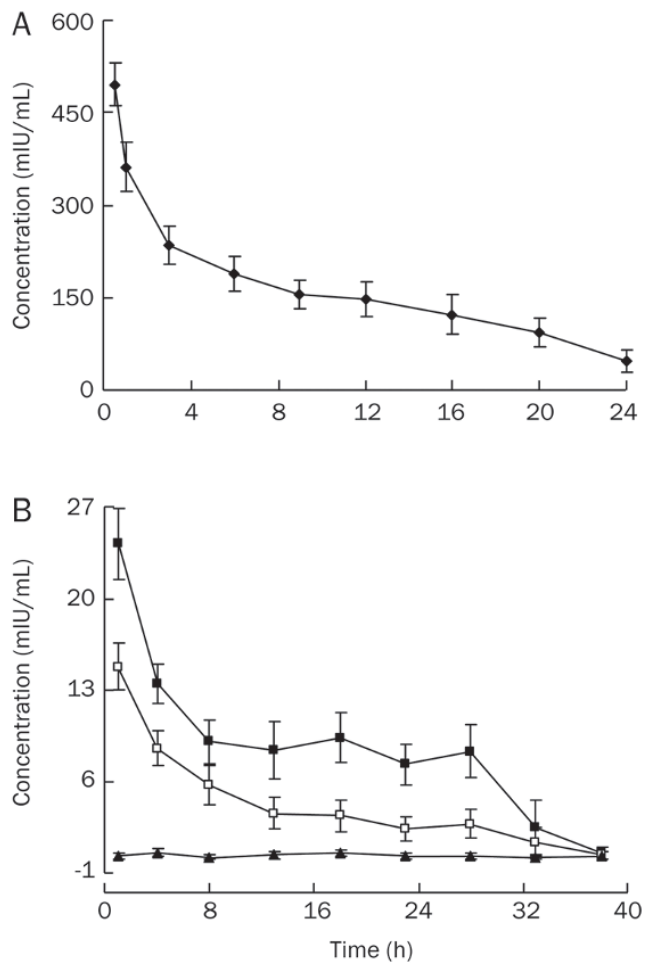

Figure 3. (A) The rhEPO plasma levels after intramuscular injection of rhEPO solution at a dose of $30000 \mathrm{IU} / \mathrm{kg}$ rhEPO in rats. (B) The rhEPO plasma levels after intramuscular injection of PLGA microspheres in rats: $10000 \mathrm{IU} \mathrm{rhEPO} / \mathrm{kg}(\square), 30000 \mathrm{IU} \mathrm{rhEPO} / \mathrm{kg}(\mathbf{m})$ and the equivalent amount of blank PLGA microspheres without $\operatorname{rhEPO}(\mathbf{\Delta})$. Mean \pm SD. $n=6$.

of the rhEPO-loaded microspheres showed that the rhEPO plasma concentrations peaked during the first day following injection. The concentrations then decreased gradually and were maintained at a stable level from $\mathrm{d} 1$ to $\mathrm{d} 28$, approaching zero at $\mathrm{d} 38$. The plasma rhEPO concentrations were significantly higher than that of the control group at d $33(P<0.05)$. The pharmacokinetic parameters estimated from the concentration-time profiles are summarized in Table 1. The areas under the curve $\left(\mathrm{AUC}_{0-33}\right)$ were 125.42 and $384.33 \mathrm{mIU} / \mathrm{mL}$ for the microsphere formulation at the doses of $10000 \mathrm{IU}$ $\mathrm{rhEPO} / \mathrm{kg}$ and $30000 \mathrm{IU} \mathrm{rhEPO} / \mathrm{kg}$, respectively. The elimination half-life of rhEPO release from PLGA microspheres was estimated to be $8.66 \mathrm{~d}$ and $12.83 \mathrm{~d}$ for the microsphere formulations at doses of $10000 \mathrm{IU} \mathrm{rhEPO} / \mathrm{kg}$ and $30000 \mathrm{IU}$ $\mathrm{rhEPO} / \mathrm{kg}$, respectively (Table 1 ). The apparent elimination rate constant $K_{\mathrm{e}}$ was determined to be $0.08 \mathrm{~h}^{-1}$ and $0.054 \mathrm{~h}^{-1}$ for the microsphere formulations at doses of $10000 \mathrm{IU} \mathrm{rhEPO} / \mathrm{kg}$ and $30000 \mathrm{IU} \mathrm{rhEPO} / \mathrm{kg}$, respectively (Table 1 ).

The in vivo degradation of the rhEPO-loaded PLGA microspheres was observed with SEM after isolation from the injection site. The results are illustrated in Figure 4. The microspheres underwent progressive changes in surface morphology throughout the experimental period. One day after intramuscular injection, the microspheres exhibited a distorted, irregular shape (Figure 4A). The distortion of microspheres may result from microsphere shrinkage, which may be an artifact of dehydration during the sample preparation for SEM. Six days later, the microspheres began to lose their smooth surface morphology, and the number of small surface pores increased significantly (Figure 4B). The microspheres completely lost their shape and exhibited a porous bulky surface after $18 \mathrm{~d}$ (Figure 4C). Although the structure of PLGA microspheres was broken after $30 \mathrm{~d}$, the residual structure could still be observed (Figure 4D).

\section{Correlation between in vitro and in vivo data}

The data obtained from the pharmacokinetic study after the intramuscular administration of the rhEPO-loaded microspheres at a dose of $30000 \mathrm{IU} / \mathrm{kg}$ were used to develop the in vitro-in vivo correlation. A plot of the percent AUC versus the percent in vitro release is shown in Figure 5. The results illustrate a good linear regression relationship between the percent in vitro release in $\mathrm{PBS}$ at $37^{\circ} \mathrm{C}$ and percent $\mathrm{AUC}$ after intramuscular administration of the rhEPO-loaded microspheres into the rats $\left(R^{2}=0.98\right)$.

\section{In vivo pharmacodynamics}

In vivo pharmacodynamics of the rhEPO-loaded PLGA microspheres were examined based on elevated hemoglobin (HGB) concentrations and RBCs in male SD rats. The results are shown in Figure 6. RBCs and the HGB concentration reached a peak at $3.0 \mathrm{~h}$ after a single injection of the rhEPO solution at a dose of $30000 \mathrm{IU} / \mathrm{kg}$. Both levels declined rapidly to baseline at approximately $24 \mathrm{~h}(P<0.05)$ (Figure 6A and 6B). On the other hand, the RBCs and HGB concentration gradually increased to peaks at $\mathrm{d} 5$ after a single injection of the rhEPOloaded microspheres (Figure 6C and 6D). Then, the peak concentrations were maintained for approximately $10 \mathrm{~d}$ and subsequently decreased slowly toward baseline. The microsphere formulations at doses of $10000 \mathrm{IU} \mathrm{rhEPO} / \mathrm{kg}$ and $30000 \mathrm{IU}$ $\mathrm{rhEPO} / \mathrm{kg}$ were sufficient to significantly increase RBCs and HGB concentrations for over 22 and $28 \mathrm{~d}$, respectively $(P<0.05)$.

\section{Acute toxicity and immunogenicity of rhEPO-loaded microsphere formulations}

A single injection of microspheres at different dosages did not induce any acute reactions or reduce body weight during the two-week observation period. No animals died.

The immunogenicity of rhEPO released from the microspheres was evaluated by measuring anti-rhEPO antibodies. A denatured rhEPO-HSA mixture and blank microspheres (without rhEPO) were used as controls. Anti-rhEPO antibodies from anti-sera taken at week 6 was analyzed by ELISA. The results are shown in Figure 7. The anti-rhEPO IgG titer induced by the rhEPO-loaded PLGA microspheres was comparable to the titer induced by the rhEPO solution $(P>0.05)$. The titers induced by the rhEPO-loaded PLGA microspheres and the rhEPO solution were significantly higher than that elicited by the blank control $(P<0.05)$ and significantly lower than that elicited by the denatured rhEPO-HSA mixture $(P<0.01)$. 

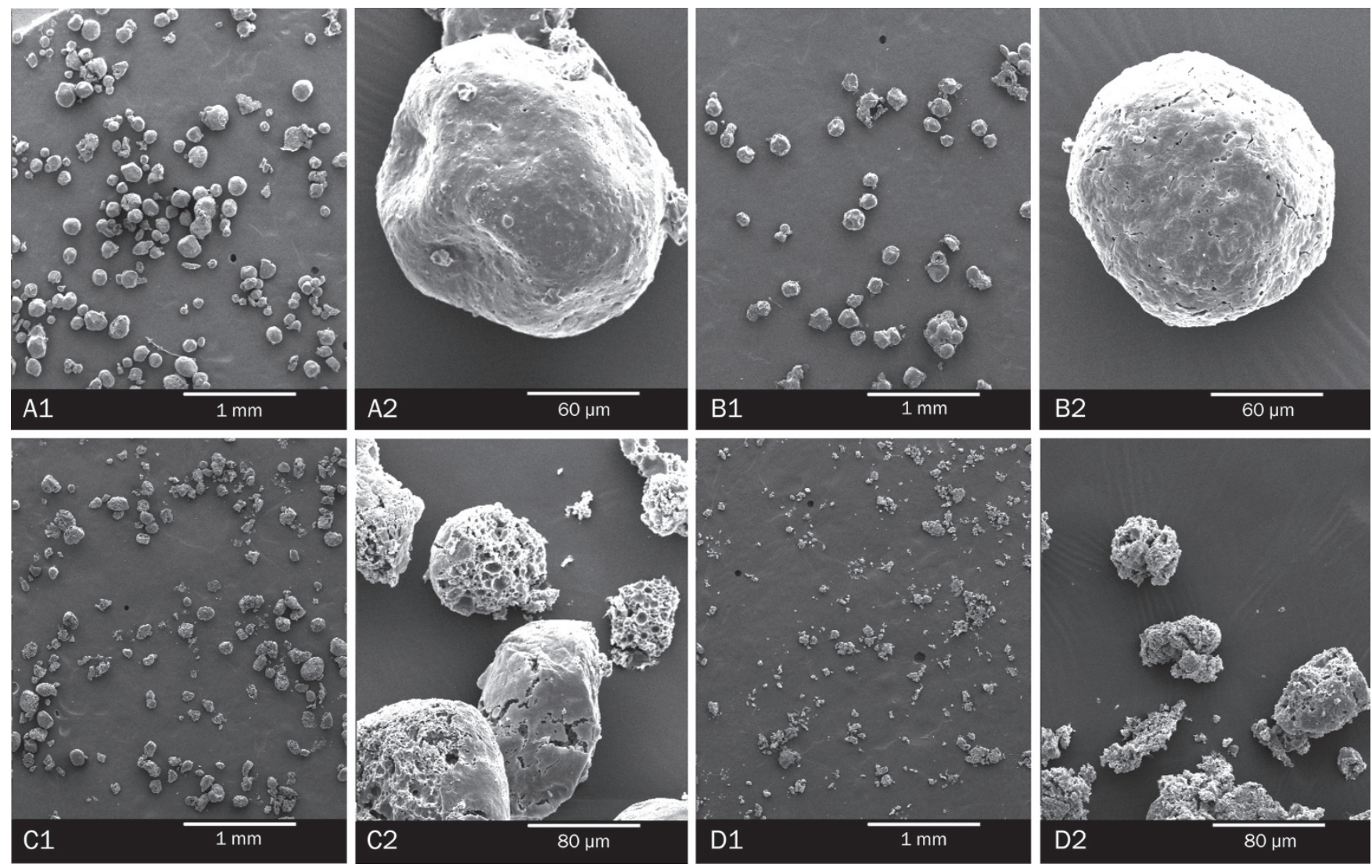

Figure 4. Scanning electron micrographs of PLGA microspheres recovered from injection site at different time. (A) One day after intramuscular injection; (B) Six days after injection; (C) Eighteen days after injection; (D) Thirty days after injection.

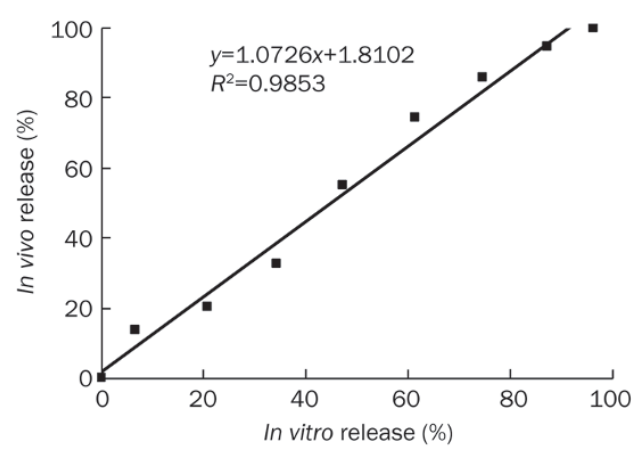

Figure 5. Correlation of in vitro and in vivo release of rhEPO from the rhEPO-loaded PLGA microsphere.

\section{Discussion}

In the present study, rhEPO-loaded PLGA microspheres were successfully prepared using a modified s/o/w emulsion solvent evaporation method. The microspheres were spherical in shape and had smooth surfaces under an electron microscope. The rhEPO recovered from PLGA microspheres was identical to that before encapsulation in our previous study ${ }^{[14]}$. An in vitro release test showed that rhEPO was released from PLGA microspheres following ideal zero-order kinetics for approxi- mately $30 \mathrm{~d}$ after a low initial burst, and this release was nearly complete after $33 \mathrm{~d}$. In SD rats, the pharmacokinetic parameters $t_{1 / 2}$ and $\mathrm{AUC}_{0-\mathrm{t}}$ for the rhEPO microsphere formulation were significantly higher than for the rhEPO solution. The elimination half-life $t_{1 / 2}$ was 21.7 and 32.1 times higher for rhEPO in microspheres than in solution at $10000 \mathrm{IU} \mathrm{rhEPO} / \mathrm{kg}$ and $30000 \mathrm{IU} \mathrm{rhEPO} / \mathrm{kg}$, respectively. The relative bioavailability of the rhEPO released from PLGA microspheres was approximately 2.0 times that of the rhEPO solution. The rhEPO plasma concentration was sustained for $33 \mathrm{~d}$, which was similar to the in vitro release data. The apparent elimination rate constant $K_{\mathrm{e}}$ was approximately 21.9 and 32.4 times lower for the rhEPO microspheres than the rhEPO solution at $10000 \mathrm{IU} \mathrm{rhEPO} / \mathrm{kg}$ and $30000 \mathrm{IU} \mathrm{rhEPO} / \mathrm{kg}$, respectively.

Two possible mechanisms may be involved to explain the release of rhEPO from PLGA microspheres. Protein dissolution/diffusion from the microspheres and/or erosion of the matrix due to degradation/dissolution of PLGA polymers ${ }^{[15,16]}$ may be responsible. Because the degradation/dissolution of PLGA takes quite a long time, the polymer backbone probably retains integrity without significant degradation/dissolution at the early release stage. As a result, drug release from the microspheres is most likely controlled by a dissolution/diffusion mechanism during the early release stage. Polymer erosion would increase drug diffusion through the polymer 

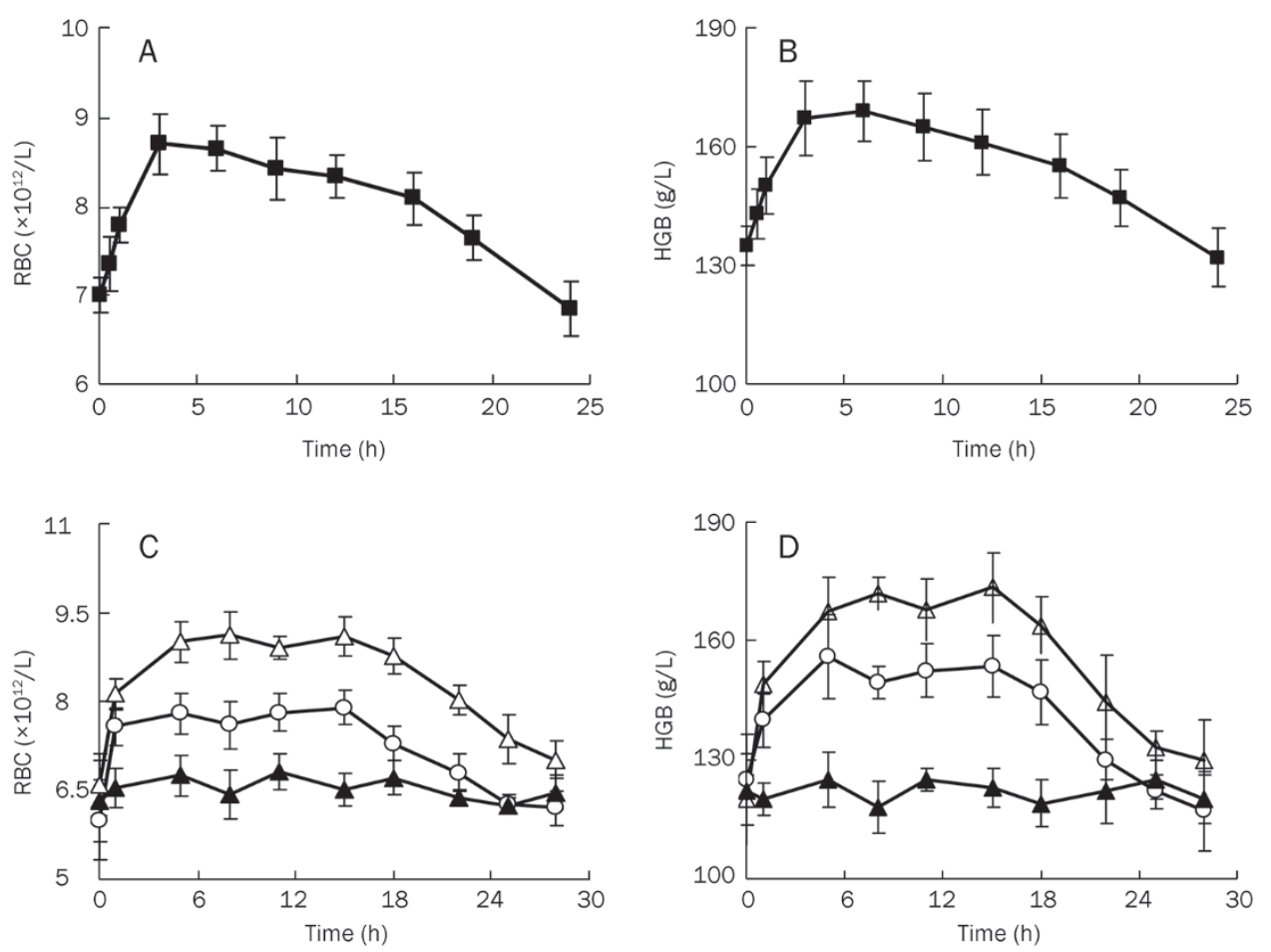

Figure 6. Profiles of red blood cell (RBC) and hemoglobin (HGB) levels in SD rats after intramuscular injection of different rhEPO formulation. (A) and (B): $3 \times 10^{4} \mathrm{IU}$ $\mathrm{rhEPO} / \mathrm{kg}$ in solution; (C) and (D): $1 \times 10^{4}$ IU/kg rhEPO within the rhEPO-loaded microspheres $(\mathrm{O}), 3 \times 10^{4} \mathrm{IU} / \mathrm{kg}$ rhEPO within the rhEPO-loaded microspheres $(\Delta)$ and blank microspheres without $\operatorname{rhEPO}(\boldsymbol{\Delta})$. Mean \pm SD. $n=6$.

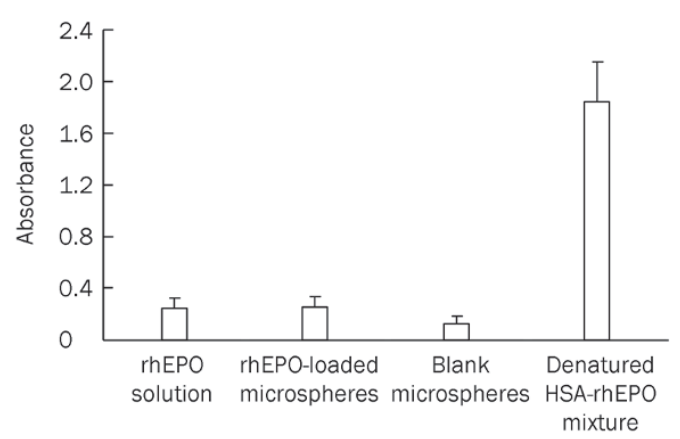

Figure 7. ELISA analysis of anti-EPO IgG within the serum from SD rats at 6 th week after subcutaneous injection of various formulations. The serum samples were diluted $1 / 200$ before ELISA analysis. Mean \pm SD. $n=5$.

matrix and increase the size of aqueous polymer pores, which facilitate the release of the entrapped proteins from the interior of PLGA microspheres ${ }^{[17]}$. We found that erosion of the polymer surface and bulk matrix occurred at later release stages in our system. Matrix erosion may contribute to rhEPO release from PLGA microspheres. However, the release rate of rhEPO from PLGA microspheres in vivo was not obviously faster than the in vitro release rate, although polymer degradation was generally faster in vivo than in vitro due to the presence of enzymes and other biological factors ${ }^{[18]}$. These results suggest that the release of rhEPO from the PLGA microspheres was not primarily controlled by matrix erosion but rather by a dissolution/diffusion mechanism.

The in vitro and in vivo correlation (IVIVC) is an important issue for parenteral biodegradable depot systems as well as for oral dosage forms ${ }^{[18,19]}$. A proper IVIVC can be used to predict an in vivo release profile for parenteral biodegradable depot systems with the aid of an in vitro release profile conducted in PBS. For erosion-controlled sustained-release depot systems, the release of PLGA-based microspheres is often slower in vitro than in vivo. Thus, it is difficult to establish a good IVIVC for erosion-controlled depot systems. It has been reported that a proper IVIVC can usually be obtained for diffusioncontrolled sustained-release depot systems ${ }^{[20]}$. The release of rhEPO from the PLGA microspheres was controlled mainly by dissolution/diffusion in our depot system. Therefore, good IVIVC for rhEPO-loaded PLGA microspheres was obtained, and the results confirmed our speculations.

The pharmacodynamic studies indicated that the rhEPOloaded PLGA microspheres could maintain elevated RBC and HGB levels over $28 \mathrm{~d}$, which was similar to the drug residence time in plasma. These results suggest that the released rhEPO was biologically active during sustained release.

PLGA microspheres have enhanced the immunogenicity of vaccines ${ }^{[21]}$. Whether the microsphere formulation increases the immunogenicity of rhEPO was investigated. An antirhEPO antibody was clearly elicited by the rhEPO solution in rats. The rat and human EPO sequences differ by thirtyfive amino acids ${ }^{[22]}$, and thus, rats may generate anti-rhEPO antibodies. The rhEPO released from the PLGA microspheres elicited a comparable amount of anti-rhEPO antibody to the solution formulation and significantly less antibody than was elicited from the denatured rhEPO-HAS mixture. These results suggest that the immunogenicity of the encapsulated rhEPO did not increase when the rhEPO-loaded microspheres were given as a depot. Our results are similar to a previous report on a sustained-release formulation of human growth hormone ${ }^{[23]}$. 
In conclusion, the PLGA microspheres containing rhEPO were successfully prepared with an $\mathrm{s} / \mathrm{o} / \mathrm{w}$ emulsion solvent evaporation method. An in vitro release test showed that rhEPO was released from PLGA microspheres following nearly zero-order kinetics for approximately $30 \mathrm{~d}$. In vivo, after intramuscular injection, the plasma concentration of rhEPO reached a maximum at $\mathrm{d} 1$, decreased gradually, and was maintained at a near constant level for approximately 4 weeks. Furthermore, the release of rhEPO from the PLGA microspheres was found to be controlled primarily by a dissolution/diffusion mechanism. A good linear correlation was also observed between the in vitro and the in vivo release data, suggesting that in vitro release data may be used to predict in vivo drug release performance. A single injection of rhEPOloaded PLGA microspheres in SD rats resulted in elevated hemoglobin and red blood cell concentrations for over $28 \mathrm{~d}$. Moreover, the immunogenicity of rhEPO released from this formulation, as assessed by the titer of anti-rhEPO antibody from SD rats, was comparable to that of the unencapsulated protein. No toxic effects were observed in an acute toxicity test in SD rats. Therefore, the present study demonstrates the feasibility of delivering rhEPO for approximately 1 month with injectable PLGA-based microspheres.

\section{Acknowledgements}

This project was supported by the Provincial Science and Technology Department of Hebei Province (09276418D-3) and the Science and Technology Project of the Provincial Education Department of Hebei (200645).

\section{Author contribution}

Xiang-lian ZHOU, Hui-juan DU, and Yang-yang FAN performed the main experiments; Jin-tian HE designed the experiment and drafted the manuscript; Ying WANG and Hong-xia ZHANG analyzed the data; Hui-juan DU and Yang JIANG aided in the design of the experiment and improved the manuscript.

\section{References}

1 Davis JM, Aralkawas T, Strickland TW, Yphantis DA. Characterization of recombinant human erythropoietin produced in Chinese hamster ovary cells. Biochemistry 1987; 26: 2633-8.

2 Eschbach JW, Egrie JC, Downing MR, Browne JK, Adamson JW. Correction of the anemia of end-stage renal disease with recombinant human erythropoietin. N Engl J Med 1987; 316: 73-8.

3 Markham A, Bryson H, Epoetin A. A review of its pharmacodynamic and pharmacokinetic properties and therapeutic use in non-renal applications. Drugs 1995; 49: 232-54.

4 Mundargi RC, Babu VR, Rangaswamy V, Patel P, Aminabhavi TM. Nano/micro technologies for delivering macromolecular therapeutics using poly( $D, L$-lactide-co-glycolide) and its derivatives. J Control Release 2008; 125: 193-209.

5 Pisal DS, Kosloski MP, Balu-lyer SV. Delivery of therapeutic proteins. J Pharm Sci 2010; 99: 2557-75.

6 Morlock M, Koll H, Winter G, Kissel T. Microencapsulation of rherythropoietin, using biodegradable poly( $D L$,-lactide-co-glycolide): protein stability and the effects of stabilizing excipients. Eur J Pharm
Biopharm 1997; 43: 29-36.

7 Morlock M, Kissel T, Li YX, Koll H, Winter G. Erythropoietin loaded microspheres prepared from biodegradable LPLG-PEO-LPLG triblock copolymers: protein stabilization and in-vitro release properties. J Control Release 1998; 56: 105-15.

8 Pistel KF, Bittner B, Koll H, Winter G, Kissel T. Biodegradable recombinant human erythropoietin loaded microspheres prepared from linear and star-branched block copolymers: Influence of encapsulation technique and polymer composition on particle characteristics. J Control Release 1999; 59: 309-25.

9 Geng Y, Yuan W, Wu F, Chen J, He M, Jin T. Formulating erythropoietinloaded sustained-release PLGA microspheres without protein. J Control Release 2008; 130: 259-65.

10 Morita T, Horikiri Y, Yamahara H, Suzuki T, Yoshino H. Formation and isolation of spherical fine protein microparticles through lyophilization of protein-poly(ethylene glycol) aqueous mixture. Pharm Res 2000; 17: 1367-73.

11 Morita T, Sakamura Y, Horikiri Y, Suzuki T, Yoshino H. Protein encapsulation into biodegradable microspheres by a novel s/o/ w emulsion method using poly(ethylene glycol) as a protein micronization adjuvant. J Control Release 2000; 69: 435-44.

12 Zhu G, Mallery SR, Schwendeman SP. Stabilization of proteins encapsulated in injectable poly(lactide-co-glycolide). Nat Biotechnol 2000; 18: 52-7.

13 El-Sayed YM, Niazy EM, Khidr SH. In vivo evaluation of sustainedrelease microspheres of metoclopramide hydrochloride in beagle dogs. Int J Pharm 1995; 123: 113-8.

14 He J, Feng M, Zhou X, Ma Sh, Jiang Y, Wang Y, et al. Stabilization and encapsulation of recombinant human erythropoietin into PLGA microspheres using human serum albumin as a stabilizer. Int J Pharm 2011; 416: 69-76.

15 Wischke C, Schwendeman SP. Principles of encapsulating hydrophobic drugs in PLA/PLGA microparticles. Int J Pharm 2008; 364: 298-327.

16 Sun Y, Wang J, Zhang X, Zhang Z, Zheng Y, Chen D, et al. Synchronic release of two hormonal contraceptives for about one month from the PLGA microspheres: in vitro and in vivo studies. J Control Release 2008; 129: 192-9.

17 Cohen S, Yoshioka T, Lucarelli M, Hwang LH, Langer R. Controlled delivery systems for proteins based on poly(lactic/glycolic acid) microspheres. Pharm Res 1991; 8: 713-20.

18 Zolnik BS, Burgess DJ. Evaluation of in vivo-in vitro release of dexamethasone from PLGA microspheres. J Control Release 2008; 127: 137-45.

19 Chu DF, Fu XQ, Liu WH, Liu K, Li YX. Pharmacokinetics and in vitro and in vivo correlation of huperzine A loaded poly(lactic-co-glycolic acid) microspheres in dogs. Int J Pharm 2006; 325: 116-23.

20 Van Dijkhuizen-Radersma R, Wright SJ, Taylor LM, John BA, De Groot K, Bezemer JM. In vitro/in vivo correlation for ${ }^{14} \mathrm{C}$-methylated lysozyme release from poly(ether-ester) microspheres. Pharm Res 2004; 21 : 484-91.

21 Tamber H, Johansen P, Merkle HP, Gander B. Formulation aspects of biodegradable polymeric microspheres for antigen delivery. Adv Drug Deliv Rev 2005; 57: 357-76.

22 Nagao M, Suga H, Okano M, Masuda S, Narita H, Ikura K, et al. Nucleotide sequence of rat erythropoietin. Biochim Biophys Acta 1992; 1171; 99-102.

23 Lee HJ, Riley G, Johnson O, Cleland JL, Kim N, Charnis M, et al. In vivo characterization of sustained-release formulations of human growth hormone. J Pharmacol Exp Ther 1997; 281: 1431-9. 